\title{
Acoustic Enhancement of Surface Diffusion
}

\author{
Chengping $\mathrm{Wu}{ }^{\dagger}$ Vladimir Yu. Zaitsev, ${ }^{\dagger,}$ and Leonid V. Zhigilei* ${ }^{*}{ }^{\dagger}$ \\ ${ }^{\dagger}$ Department of Materials Science and Engineering, University of Virginia, Charlottesville, Virginia 22904, United States \\ ${ }^{\ddagger}$ Institute of Applied Physics, Russian Academy of Sciences, Nizhny Novgorod, Russia
}

\section{Supporting Information}

ABSTRACT: The idea of acoustic activation of surface diffusion is explored theoretically and in atomistic simulations. It is found that a substantial diffusion enhancement by surface acoustic waves is possible via (1) transient surface straininduced modification of the diffusion barriers, (2) adiabatic variation in the surface temperature, and (3) dynamic coupling of the acoustic waves with vibrational states of adsorbed species. The approximate scaling laws describing the first two effects are established and verified in kinetic Monte Carlo simulations. The combined contribution of all three effects is studied in molecular dynamics simulations, and the conditions

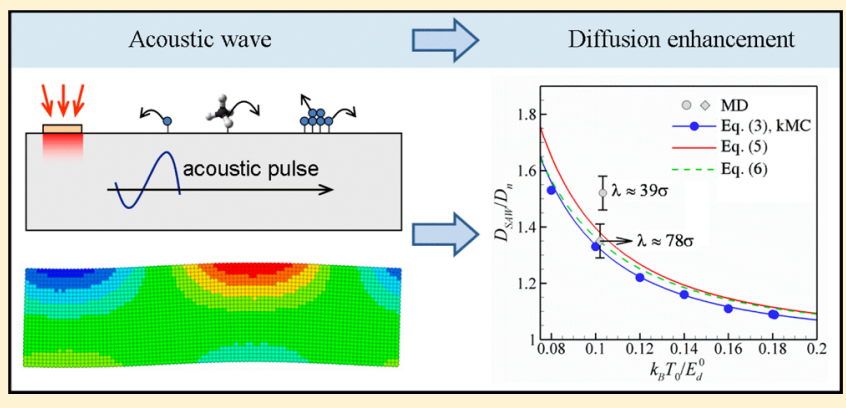
for the diffusion activation through the dynamic coupling are elucidated. The acoustic enhancement of surface diffusion provides an attractive alternative to thermal activation in thin film growth on heat-sensitive substrates.

\section{INTRODUCTION}

The ability of surface acoustic waves (SAWs), or Rayleigh waves, to propagate and transfer energy along a thin (several wavelengths deep) surface layer of substrate with little dissipation has been utilized in a number of applications ranging from nondestructive evaluation of mechanical properties and surface defects ${ }^{1-3}$ to micro- and nanoscale manipulation of particles, biomolecules, and fluid flow in microfluidics devices. ${ }^{4,5}$ At the atomic level, however, the ability of SAWs to directly affect surface mobility of individual adatoms or adsorbed molecules remains largely unexplored. The lack of attention to the acoustic activation of surface diffusion can be explained by an intuitive expectation that, due to the large frequency mismatch between SAWs and vibrational states of the adsorbed species, the possibility of dynamic coupling of SAWs to the adsorbates can be excluded, whereas periodic perturbations of surface energy landscape associated with the acoustic strain should have a negligible effect on adatom mobility. This intuitive reasoning, however, comes into conflict with emerging experimental evidence of substantial enhancement of surface catalysis ${ }^{6-11}$ and molecular desorption $^{12-17}$ by acoustic waves, as well as recent observation of SAW-induced directional motion of small atomic Au clusters on a $\mathrm{Si}$ substrate. ${ }^{18}$ These intriguing results call for a detailed analysis of the acoustic activation of atomic-level surface processes.

The results of a theoretical and computational investigation reported in this paper demonstrate that the enhancement of surface diffusion under the action of acoustic waves can reach tens and hundreds of percents. The mechanisms responsible for the enhancement of the surface diffusion of adsorbed atoms and the degree to which the diffusion can be affected/ controlled by SAWs are revealed in a systematic study that combines theoretical analysis with kinetic Monte Carlo (kMC) and molecular dynamics (MD) simulations. The prospects for the acoustic activation of surface diffusion illuminated in this study suggest an attractive alternative to the thermal activation in thin film growth on heat-sensitive substrates.

\section{MECHANISMS OF ACOUSTIC ACTIVATION OF SURFACE DIFFUSION}

The modification of surface diffusion by SAWs can be realized through the following three mechanisms: (1) modification of the diffusion barriers through transient variation of surface strain due to the SAW propagation, (2) variation of surface temperature due to the adiabatic heating and cooling associated with the fast surface deformation, and (3) dynamic coupling of the SAW to surface vibrational states of the adsorbed species. When considering the temperature dependence of the surface diffusion coefficient in the presence of a plane SAW propagating in the $x$-direction, these three effects can be represented as follows

$$
D(x, t)=D_{0} \exp \left(-\frac{E_{\mathrm{d}}(x, t)}{k_{\mathrm{B}} T(x, t)+\Delta E_{\mathrm{SAW}}}\right)
$$

Here, the SAW manifests itself via spatial and time variations of the surface temperature $T(x, t)$ and the diffusion barrier $E_{\mathrm{d}}(x, t)$, as well as via the energy of the nonthermal vibrational excitation of the adsorbates $\Delta E_{\mathrm{SAW}}$ due to the dynamic coupling with the SAW.

Received: January 25, 2013

Revised: April 5, 2013

Published: April 10, 2013 
Table 1. Values of the Diffusion Barrier $E_{\mathrm{d}}^{0}$ and Scaling Factor $\gamma$ in Equation 2 Obtained in Quasi-Static Calculations and MD Simulations (Italicized Cells) Performed for Different Values of $\sigma_{\mathrm{a}-\mathrm{s}}$ and Uniaxial Lateral Strain $e_{x x}$ Ranging from -0.04 to 0.04

$\sigma_{\mathrm{a}-\mathrm{s}} \quad 0.8 \sigma$

$E_{\mathrm{d}}^{0} / \varepsilon$

$\gamma / E_{\mathrm{d}}^{0}$
$0.9 \sigma$

1.69

1.07

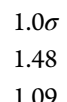

1.09

$1.0 \sigma(\mathrm{MD})$
1.48
1.03

1.03

$\begin{array}{ll}1.1 \sigma & 1.2 \sigma \\ 1.28 & 1.07 \\ 1.31 & 1.54\end{array}$

$1.3 \sigma$
0.86
1.98
$1.4 \sigma$

0.66

2.15
$1.5 \sigma$
0.47
2.64

$1.5 \sigma(M D)$

0.49

2.57
The dynamic coupling between the adsorbed species and the SAW requires proximity to the "resonance condition"; ${ }^{15}$ i.e., the frequency of the SAW should be comparable to the ones of the surface vibrational states. While this requirement makes the dynamic coupling an unlikely mechanism for sub-gigahertz SAWs typically generated with conventional interdigital transducers, recent advances in the development of photoacoustic methods for generation of SAWs with frequencies approaching $100 \mathrm{GHz}^{19-21}$ suggest that the acoustic activation of this mechanism may be feasible, particularly for physisorbed molecules weakly bound to the surface. Therefore, the contributions from all three mechanisms are analyzed below and related to the parameters of SAWs and characteristics of material/adsorbate systems.

2.1. Modification of Diffusion Barriers by Surface Strain. The effect of the modification of diffusion barriers, $E_{\mathrm{d}}(x, t)$, through transient variation of surface strain is related to the strong nonlinearity of the dependence of the diffusion coefficient on the activation barrier. Even if the surface strain in the SAW induces symmetric variation of the activation barrier around a zero-strain value, $E_{\mathrm{d}}^{0}$, the exponential dependence on $E_{\mathrm{d}}$ may still result in a strong enhancement of the diffusion coefficient. The effect of the surface strain on the diffusion barrier has been investigated in a number of quasi-static atomistic calculations. ${ }^{22-27}$ The calculations performed with density-functional theory $(\mathrm{DFT})^{23,25-27}$ and classical $\mathrm{MD}^{22,24}$ for adatom diffusion on $\mathrm{Si}$ and $\mathrm{Ge}(001),{ }^{22,26,27} \mathrm{Ag}(111){ }^{23}$ $\mathrm{GaAs}(100){ }^{25}$ and Lennard-Jonesium $(001)^{24}$ surfaces predict that surface strain on the order of $10^{-3}-10^{-2}$ can result in the variation of $E_{\mathrm{d}}$ by $5-10 \%$. Although for surfaces with complex anisotropic diffusion paths the effect of strain on surface energy landscape is complex and may depend on the strain type (biaxial or uniaxial) and direction, ${ }^{25-27}$ for a variety of material systems and surface strains up to several percents ${ }^{23-27}$ the variation of the diffusion barriers can be relatively well approximated by a linear dependence, i.e.

$$
E_{\mathrm{d}}(e) \approx E_{\mathrm{d}}^{0}+\gamma e
$$

where $e$ is the magnitude of the surface strain; $\gamma$ is the coefficient of proportionality between the diffusion barrier and surface strain; and $E_{\mathrm{d}}^{0}$ is the unperturbed diffusion barrier at $e=$ 0.

To complement the existing data on the strain dependence of the diffusion barrier and to ensure reliable interpretation of the results of MD simulations reported below, we performed a series of calculations of energy barriers for adatom diffusion on (001) surfaces of strained fcc substrates. The interatomic interactions in the substrate are described by the Lennard-Jones (LJ) potential with energy and length parameters $\varepsilon$ and $\sigma$, a cutoff function suggested in ref 28, and a cutoff distance of $3 \sigma$. The adatom-substrate interaction is described by the same potential, except for the length parameter, $\sigma_{\mathrm{a}-\mathrm{s}}$, that is varied between $0.8 \sigma$ and $1.5 \sigma$ to study the effect of the size of the adsorbate on the strain dependence of the diffusion barrier. Regardless of $\sigma_{\mathrm{a}-s}$, the adatom is assumed to have the same mass $m$ as the substrate atoms. The calculations are performed for a substrate composed of $12 \times 12 \times 12 \mathrm{fcc}$ unit cells, with periodic boundary conditions applied in $x$ and $y$ directions and free boundary conditions used in the $z$ direction. The uniaxial lateral strain $e_{x x}$ ranging from -0.04 to 0.04 (negative values correspond to compression) is imposed by changing the size of the substrate in the $x$ direction. The diffusion barrier is defined as the difference of the total energies of systems with the adatom located at the binding site $\left(E_{\mathrm{B}}\right)$ and the saddle point $\left(E_{\mathrm{S}}\right)$, i.e., $E_{\mathrm{d}}=E_{\mathrm{S}}-E_{\mathrm{B}}$. The values of $E_{\mathrm{S}}$ and $E_{\mathrm{B}}$ are evaluated by fixing the lateral position $(x, y)$ of the adatom at the binding site or the saddle point of the (001) surface and allowing the system to relax (to achieve the energy minimum) while allowing the adatom to only move in the $z$ direction. The values of $E_{\mathrm{d}}$ are found to follow the linear scaling with strain given by eq 2 , and the results of the calculations are summarized in Table 1.

The diffusion barriers evaluated by an alternative method, based on the results of direct MD simulations of adatom diffusion, are also listed in Table 1 for $\sigma_{\mathrm{a}-s}=\sigma$ and $\sigma_{a-s}=1.5 \sigma$. In this case, several series of simulations are performed at different temperatures. At each temperature, $N=120$ independent adatom trajectories with a length of $8400 \tau_{0}$ are obtained, where $\tau_{0}=\left(m \sigma^{2} / \varepsilon\right)^{1 / 2}$ (for reference, the period of adatom vibrations is $\sim 1.2 \tau_{0}$ for $\sigma_{\mathrm{a}-s}=\sigma$ and $\sim 2.8 \tau_{0}$ for $\sigma_{\mathrm{a}-s}=$ $1.5 \sigma)$. The diffusion coefficients are determined from time dependences of the mean square displacements of the adatoms, $\left\langle\Delta \vec{r}(t)^{2}\right\rangle \equiv\left[1 /\left(N \times N_{t}\right)\right] \sum_{i=1}^{N} \sum_{j=1}^{N_{t}}\left[\vec{r}_{i}\left(t+t_{j}\right)-\vec{r}_{i}\left(t_{j}\right)\right]^{2}=4 D t$, where $\vec{r}_{i}(t)$ are "unfolded" true adatom positions not affected by the periodic boundary conditions. The diffusion barrier is then obtained from the Arrhenius plot for the temperature dependence of the diffusion coefficient. A good agreement between the values of $E_{\mathrm{d}}^{0}$ and $\gamma$ obtained in quasi-static and dynamic calculations supports the notion of the involvement of the motions of substrate atoms in the adatom jumps, accounted for by the relaxation of the substrate in the evaluation of the saddle point energy in the quasi-static calculations. Contrary to the assessment of ref 24 , a procedure where no relaxation of the substrate is done in the calculation of the saddle point energy leads to a substantial overestimation (up to $60 \%$ for $\sigma_{\mathrm{a}-s}=$ $1.5 \sigma$ ) of the diffusion barriers as compared to the MD simulations.

2.2. Temperature Variation Due to the Adiabatic Heating and Cooling. Turning to the second mechanism of the acoustic modification of the diffusion coefficient given by eq 1 , the variation of surface temperature $T(x, t)=T_{0}+\Delta T(x, t)$ induced by the SAW can be evaluated by considering the nearly adiabatic transient acoustic deformation of the surface region. For the reversible adiabatic heating and cooling, $\Delta T / T_{0}=$ $-K_{\mathrm{b}} \alpha e_{i i}^{\mathrm{SAW}} / c_{V}{ }^{29}$ where $K_{\mathrm{b}}$ is the bulk elastic modulus; $\alpha$ is the thermal expansion coefficient; $\mathcal{c}_{V}$ is the specific heat capacity at constant volume; and $e_{i i}^{\mathrm{SAW}}$ is the dilatation caused by the acoustic deformation. Considering a SAW running along the [100] direction on a (001) surface of an fcc LJ crystal, the surface strain in the direction of the SAW propagation can be described as $e_{x x}(x, t)=e_{0} \sin (k x-\Omega t)$, where $e_{0}$ is the amplitude; $\Omega=2 \pi c / \lambda$ is the angular frequency; $k=2 \pi / \lambda$ is the 


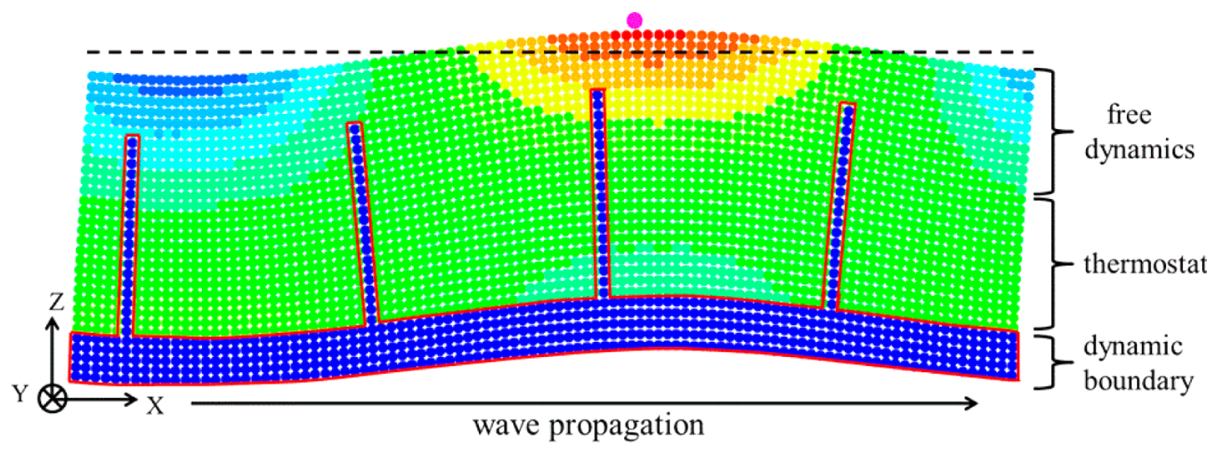

Figure 1. Computational system used in MD simulations of adatom diffusion on a substrate in the presence of a SAW propagating along the $x$ direction. The blue atoms in the region outlined by the red solid lines are displaced according to the analytical expressions for the desired Rayleigh wave. The purple atom is the adatom diffusing on the surface. The other atoms are colored by the instantaneous value of local strain. The atoms in the topmost 12 monolayers undergo unconstrained free dynamics, and the atoms of the following 14 monolayers are coupled with a thermostat to keep a constant temperature of the system. The black dashed line marks the position of the free surface in the absence of SAW, and the black arrow shows the wave propagation direction. The dynamics of adatom diffusion in one of the MD simulations can be viewed in the Supporting Information, movie jp400884d_si_004.avi.

wave vector; $c$ is the propagation velocity; and $\lambda$ is the wavelength of the SAW. The dilatation in the vicinity of the free surface is defined by two strain components with opposite signs, $e_{i i}^{\mathrm{SAW}}=e_{x x}+e_{z z}=\left(\left(C_{11}-C_{12}\right) / C_{11}\right) e_{x x}$, and is smaller than the one for the bulk wave, $e_{i i}=e_{x x}$. Using the thermoelastic parameters of the LJ material, the dilatation and temperature variations can be expressed as $e_{i i}^{\text {SAW }} \approx 0.43 e_{x x}$ and $\Delta T / T_{0} \approx$ $-1.56 e_{x x}$.

The adiabatic temperature variation can also be directly studied in $\mathrm{MD}$ simulations performed for the $\mathrm{LJ}$ system discussed above, where the running SAW is generated and maintained as illustrated in Figure 1. The computational system consists of 31 atomic planes in the $z$ direction and 24 atomic planes ( $12 \mathrm{fcc}$ unit cells) in the $y$ direction and has a size equal to the wavelength $\lambda$ of the SAW in the $x$ direction. The periodic boundary conditions are applied in $x$ and $y$ directions that are parallel to the (001) free surface of the system. Prior to the introduction of the SAW, the substrate is equilibrated at a desired temperature. The SAW is then initialized by assigning displacements and additional velocities to all atoms in the substrate according to the analytical expressions for the Rayleigh wave in an fcc crystal. ${ }^{30}$ The SAW is then maintained by applying the dynamic boundary conditions to the bottom five atomic planes and four partial vertical planes, where the atomic displacements follow the analytical expressions for the Rayleigh wave. ${ }^{30}$ The dynamic boundary condition suppresses the generation of higher harmonics that distort the shape of the SAW, particularly in systems with small $\lambda$. To avoid heating of the substrate due to the dissipation of the SAW, the temperature of 14 atomic layers adjacent to the bottom part of the dynamic boundary is maintained with the Berendsen thermostat method, ${ }^{31}$ with the atoms subject to the dynamic boundary condition excluded from the thermostat region. The atoms in the 12 topmost atomic layers of the substrate undergo unconstrained free dynamics. The instantaneous local temperature is calculated from kinetic energies of atoms in rows along the $y$ direction and the neighboring rows. The collective velocity due to the acoustic wave propagation is subtracted from atomic velocities used in the temperature calculation.

The results of the calculation of the instantaneous strain and temperature distributions in a MD simulation performed for a running SAW with the lateral surface strain amplitude $e_{0}=$ 0.048 and the wavelength $\lambda \approx 78 \sigma$ (50 fcc unit cells) are shown in Figure 2. To reduce thermal noise in the strain and temperature distributions, the results are averaged over 138
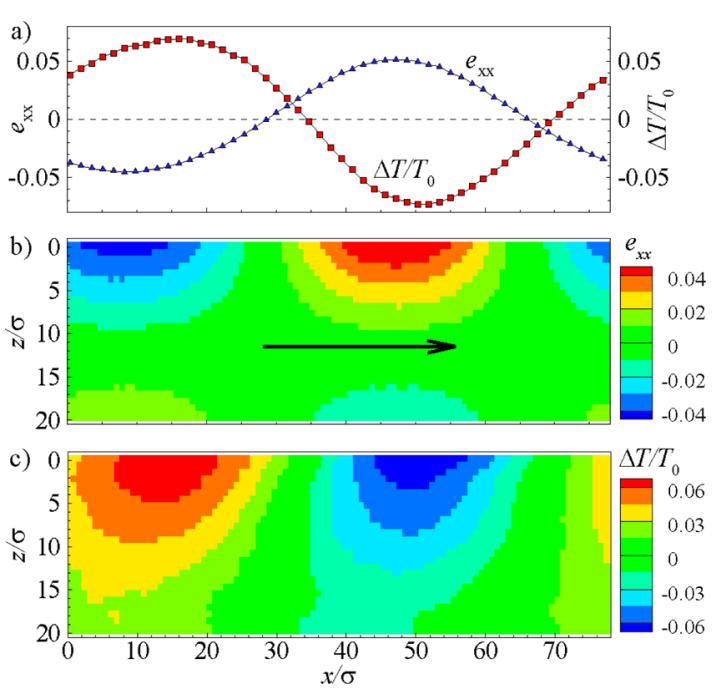

Figure 2. Instantaneous strain and temperature distributions in a $\mathrm{MD}$ simulation performed for a running SAW with $e_{0}=0.048$ and $\lambda \approx 78 \sigma$. The average temperature $T_{0}$ of the surface is maintained close to the thermostat temperature $T_{\mathrm{th}}=0.15 \varepsilon / k_{\mathrm{B}}$. To reduce thermal noise in the strain and temperature distributions, the results are averaged over 138 periods of wave propagation. The strain and temperature in the top surface monolayer are shown in (a) by blue delta and red square symbols, respectively. The contour plots in (b) and (d) show the strain and temperature distributions in the subsurface region of the substrate. The black arrow in (b) shows the direction of the wave propagation. The time evolution of the surface strain and temperature profiles in this simulation can be viewed in Supporting Information, movies jp400884d_si_002.avi and jp400884d_si_003.avi.

periods of the wave. The profile of the surface strain shown in Figure 2a follows closely the expected distribution of $e_{x x}(x, t)=$ $e_{0} \sin (k x-\Omega t)$ at a fixed time $t$, whereas the profile of the surface temperature exhibits a small phase shift from the expected antiphase variation with strain. The phase shift and the asymmetry of the temperature distribution in Figure $2 \mathrm{c}$ are related to deviations from perfectly adiabatic thermoelastic heating and cooling conditions in the case of high-frequency SAWs. The phase shift decreases with increasing $\lambda$ and, even for 
the small values of $\lambda$ used in this work, is found to have little effect on surface diffusion in kMC simulations. Therefore, the antiphase temperature and strain variations, i.e., $\Delta T / T_{0} \approx$ $-\theta e_{x x}(x, t)$, are assumed in further discussion. The value of $\theta \approx$ 1.49 , obtained from about $7.2 \%$ magnitude of $\Delta T / T_{0}$ variation in Figure 2 , is in a fairly good agreement with the value of $\theta \approx$ 1.56 estimated above based on thermoelastic properties of the substrate material.

2.3. Diffusion Enhancement via Diffusion Barrier and Adiabatic Temperature Variations. With the approximations of the linear scaling of the diffusion barrier, $E_{\mathrm{d}}(x, t) \approx E_{\mathrm{d}}^{0}+$ $\gamma e_{x x}(x, t)$, and surface temperature, $\Delta T(x, t) / T_{0} \approx-\theta e_{x x}(x, t)=$ $-\left[\gamma_{T} / E_{\mathrm{d}}^{0}\right] e_{x x}(x, t)$ (where $\gamma_{\mathrm{T}}=\theta E_{\mathrm{d}}^{0}$ is introduced for convenience), the combined effect of the adiabatic temperature variation and the modification of the diffusion barriers can be evaluated by spatial and time integration of eq 1

$$
\begin{aligned}
D_{\mathrm{SAW}}= & \frac{1}{\lambda \tau} \int_{0}^{\lambda} \mathrm{d} x^{\prime} \int_{0}^{\tau} \mathrm{d} t^{\prime} \\
& D_{0} \exp \left(-\frac{E_{\mathrm{d}}^{0}+\gamma e_{x x}\left(x^{\prime}, t^{\prime}\right)}{k_{\mathrm{B}}\left[T_{0}+\Delta T\left(x^{\prime}, t^{\prime}\right)\right]}\right)
\end{aligned}
$$

where $\tau$ is the period of SAW. For small $\Delta T / T_{0}$, an approximation $1 /\left(1+\Delta T / T_{0}\right) \approx 1-\Delta T / T_{0}$ can be used, and the above equation can be rewritten as

$$
\begin{aligned}
& D_{\text {SAW }}=\frac{1}{\lambda \tau} \int_{0}^{\lambda} \mathrm{d} x^{\prime} \int_{0}^{\tau} \mathrm{d} t^{\prime} \\
& D_{0} \exp \left(-\frac{\left\{E_{\mathrm{d}}^{0}+\gamma e_{x x}\left(x^{\prime}, t^{\prime}\right)\right\}\left\{1+\left[\gamma_{\mathrm{T}} / E_{\mathrm{d}}^{0}\right] e_{x x}\left(x^{\prime}, t^{\prime}\right)\right\}}{k_{\mathrm{B}} T_{0}}\right)
\end{aligned}
$$

By neglecting the second-order term $\sim e_{x x}^{2}$ in the numerator of the exponent and considering an oscillating surface strain induced by SAW, $e_{x x}(x, t)=e_{0} \sin (k x-\Omega t)$, the diffusion coefficient can be expressed as

$$
\begin{aligned}
D_{\text {SAW }}= & \frac{D_{n}\left(T_{0}\right)}{(2 \pi)^{2}} \int_{0}^{2 \pi} \mathrm{d} x^{\prime} \int_{0}^{2 \pi} \mathrm{d} t^{\prime} \\
& \exp \left(-\frac{\gamma_{\text {eff }} e_{0} \sin \left(x^{\prime}-t^{\prime}\right)}{k_{\mathrm{B}} T_{0}}\right) \\
= & D_{n}\left(T_{0}\right) f\left(\gamma_{\text {eff }} e_{0} / k_{\mathrm{B}} T_{0}\right)
\end{aligned}
$$

where $D_{n}\left(T_{0}\right)=D_{0} \exp \left(-E_{\mathrm{d}}^{0} / k_{\mathrm{B}} T_{0}\right)$ is the diffusion coefficient in the absence of a SAW and $\gamma_{\text {eff }}=\gamma+\gamma_{\mathrm{T}}$ is the effective scaling factor that reflects the additive contributions of the diffusion barrier and temperature variations to the diffusion enhancement. For positive values of $\gamma$, such as the ones listed in Table 1 , the effect of the temperature variation acts synergistically with the diffusion barrier modification given by eq 2 ; i.e., the compressive strain $\left(e_{x x}<0\right)$ reduces the diffusion barrier $\left(E_{\mathrm{d}}-\right.$ $\left.E_{\mathrm{d}}^{0}<0\right)$, while the simultaneous temperature increase $(\Delta T>0)$ further facilitates diffusion. Interestingly, the diffusion enhancement due to the transient variation of the diffusion barrier and temperature does not depend on the wavelength of the SAW but is only a function of $\gamma_{\text {eff }} e_{0} / k_{\mathrm{B}} T_{0}$.

The diffusion enhancement factor $D_{\mathrm{SAW}} / D_{n}$ predicted by eq 5 is illustrated in Figure 3 for a range of parameters that may be relevant to practical applications. The value of $\gamma_{\text {eff }}$ is intrinsic to a specific system and defines the limits of the diffusion enhancement that can be achieved at practically relevant values of $T_{0}$ and $e_{0}$. Since the temperature $T_{0}$ that ensures a $D_{n}$ that is sufficiently high to meet the requirements of a given application

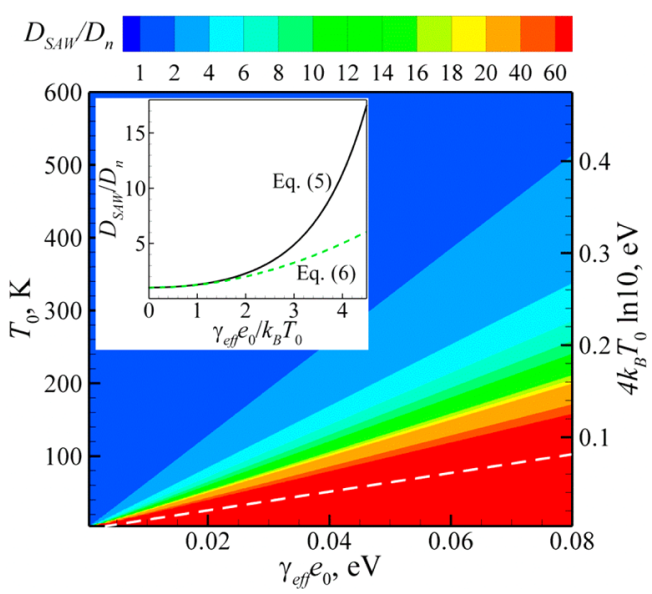

Figure 3. Diffusion enhancement factor $D_{\mathrm{SAW}} / D_{n}$ as a function of $\gamma_{\mathrm{eff}} e_{0}$ and $T_{0}$ obtained by numerical integration of eq 5 . The inset shows the diffusion enhancement as a function of $\left(\gamma_{\text {eff }} e_{0}\right) /\left(k_{\mathrm{B}} T_{0}\right)$ predicted by eqs 5 and 6 . The scale on the right side of the contour plot shows the values of the diffusion barrier $E_{\mathrm{d}}^{0 *}=4 k_{\mathrm{B}} T_{0} \ln 10$ that ensure an adatom jump rate of $r^{*} \sim 0.1-1 \mathrm{~ns}^{-1}$ at the corresponding substrate temperature $T_{0}$. The white dashed line in the contour plot corresponds to $\gamma_{\mathrm{eff}} e_{0}=E_{\mathrm{d}}^{0 *}$.

is determined by the magnitude of $E_{\mathrm{d}}^{0}$, it is actually the value of $\gamma_{\text {eff }} / E_{\mathrm{d}}^{0}$ that controls the diffusion enhancement for a given system. Assuming that an adsorbate makes one successful jump per $10^{4}$ "attempts" (jump rate $r^{*} \sim 0.1-1 \mathrm{~ns}^{-1}$ ) as an example, a complementary scale is shown on the right side of Figure 3 to illustrate the diffusion barriers $E_{\mathrm{d}}^{0 *}=4 k_{\mathrm{B}} T_{0} \ln 10$ that ensure $r^{*}$ at $T_{0}$. The corresponding natural limit of $\gamma_{\mathrm{eff}} e_{0}<E_{\mathrm{d}}^{0 *}$ is also outlined by a dashed line in Figure 3. The upper limit for $e_{0}$ is determined by the onset of surface damage ${ }^{2,3}$ and is on the order of $10^{-2}$.

To evaluate the approximate functional dependence of the diffusion enhancement factor $D_{\mathrm{SAW}} / D_{n}$ on $\gamma_{\mathrm{eff}} \mathrm{e}_{0} / k_{\mathrm{B}} T_{0}$, the exponent in eq 5 can be expanded with respect to the small value of $\gamma_{\text {eff }} e_{x x} / k_{\mathrm{B}} T_{0}$, i.e., $\exp \left(-\gamma_{\mathrm{eff}} e_{x x} / k_{\mathrm{B}} T_{0}\right) \approx 1-\gamma_{\mathrm{eff}} e_{x x} / k_{\mathrm{B}} T_{0}+$ $\left(\gamma_{\text {eff }} e_{x x} / k_{\mathrm{B}} T_{0}\right)^{2} / 2$. After the spatial and time averaging in eq 5 , this approximation yields the following scaling of the diffusion enhancement

$$
D_{\mathrm{SAW}} / D_{n} \approx 1+\frac{1}{4} \frac{\gamma_{\mathrm{eff}}^{2}}{\left(k_{\mathrm{B}} T_{0}\right)^{2}} e_{0}^{2}
$$

Thus, the diffusion enhancement can be expected for any sign of $\gamma_{\text {eff }}$ and exhibits quadratic scaling with $\gamma_{\text {eff }}$ and $e_{0}$. The relative change of the diffusion coefficient also scales as $T_{0}^{-2}$ and is larger for lower $T_{0}$, although the absolute change of the diffusion coefficient is larger at higher $T_{0}$ due to the Arrhenius temperature dependence of $D_{n}\left(T_{0}\right)$. As can be seen from the inset in Figure 3, the discrepancy between the diffusion enhancement predicted by eqs 5 and 6 is increasing at low temperatures (large $\gamma_{\mathrm{eff}} e_{0} / k_{\mathrm{B}} T_{0}$ ) due to the decreasing accuracy of the truncated Taylor expansion used in the derivation of eq 6.

The accuracy of the approximations used in the derivation of eqs 5 and 6 can be evaluated by performing one-dimensional (1D) kMC simulations of surface diffusion in the presence of a SAW. In kMC simulations, the microscopic kinetics of surface diffusion is reproduced by moving surface adatoms between the adjacent equilibrium sites with probability that is the product of the time step $\Delta t$ and the adatom jump rate, i.e., $p=R_{0}$ 
$\exp \left[-E_{\mathrm{d}}(x, t) / k_{\mathrm{B}} T(x, t)\right] \times \Delta t$, where $R_{0}$ is the so-called attempt frequency related to the vibrational frequency of a surface adatom and chosen based on the value of $D_{0}$ predicted in MD simulations. The time step is chosen to ensure that $p<1$ and $\Delta t$ is at least 1 order of magnitude smaller than the period of SAW. The kMC simulations are performed under assumption of linear strain dependences of the diffusion barriers, $E_{\mathrm{d}}(x, t)=$ $E_{\mathrm{d}}^{0}+\gamma e_{x x}(x, t)$, and surface temperature, $T(x, t)=T_{0}-$ $T_{0} \theta e_{x x}(x, t)$, with surface strain associated with a running SAW introduced as $e_{x x}(x, t)=e_{0} \sin (k x-\Omega t)$. The local asymmetry of the energy barriers related to the gradient of the acoustic strain is neglected, and the adatom has equal probability to jump to the left and to the right at any given time. The parameters of the simulations are chosen to match those determined in MD simulations performed for $\sigma_{\mathrm{a}-\mathrm{s}}=\sigma$ and $\sigma_{\mathrm{a}-\mathrm{s}}=1.5 \sigma$, with corresponding values of $E_{\mathrm{d}}^{0}$ and $\gamma$ taken from the italicized cells of Table 1 and $\theta=1.49$ used to describe the acoustically induced temperature variations. The distance between the equilibrium sites in $1 \mathrm{D} \mathrm{kMC}$ simulations is chosen to be one-half of the fcc lattice constant to allow for quantitative comparison with the results of $\mathrm{MD}$ simulations of adatom diffusion on a (001) surface of an fcc crystal. The total of 50000 independent $1000 \Delta t$-long trajectories are generated in $\mathrm{kMC}$ simulations and, similarly to the $\mathrm{MD}$ simulations discussed above, the diffusion coefficient is calculated from the time dependence of the mean square displacement of the adatoms, $\left\langle\Delta x(t)^{2}\right\rangle=2 D t$.

The diffusion enhancement found in $\mathrm{kMC}$ simulations and shown in Figure 4 is consistently below the results of the numerical integration of eq 5 . This is not surprising since both approximations used in the derivation of eq 5 , the expansion $1 /$ $\left(1+\Delta T / T_{0}\right) \approx 1-\Delta T / T_{0}$ and the neglect of a second-order term $\sim e_{x x}^{2}$ in the numerator of the exponent, contribute to overestimation of the diffusion enhancement. The deviations, however, are relatively small in the whole range of temperatures considered in the calculations. This suggests that the approximations, which allowed us to describe the combined effect of the adiabatic temperature variation and the modification of the diffusion barriers in a uniform manner, through the introduction of $\gamma_{\text {eff }}$, are reasonable. The discrepancy between the predictions of eq 5 and eq 6 in Figure 4 is also relatively small and, similarly to the inset of Figure 3, increases with decreasing $T_{0}$.

2.4. Dynamic Coupling between Adsorbates and SAWs. Finally, we turn to the third mechanism of the acoustic enhancement of surface mobility, the dynamic coupling between the adsorbed species and SAW leading to a nonthermal energy transfer to the adsorbate. The effect of dynamic coupling is represented in eq 1 as an extra energy $\Delta E_{\text {SAW }}$ added to the thermal energy of the adsorbates. Under conditions when the direct resonant coupling of the acoustic wave to the surface-adsorbate bond is prevented by the high frequency of individual vibrational surface modes, the energy transfer is likely to be a multistep process involving anharmonic dissipation of the energy of the acoustic wave to the highfrequency vibrations. A rigorous theoretical treatment of this process is difficult, and as the first step, we evaluate the contribution of the dynamic coupling to the diffusion enhancement by comparing the predictions of the kMC simulations discussed above with the results of MD simulations, which naturally account for the dynamic coupling, as well as the other two mechanisms that are reproduced in $\mathrm{kMC}$ simulations.

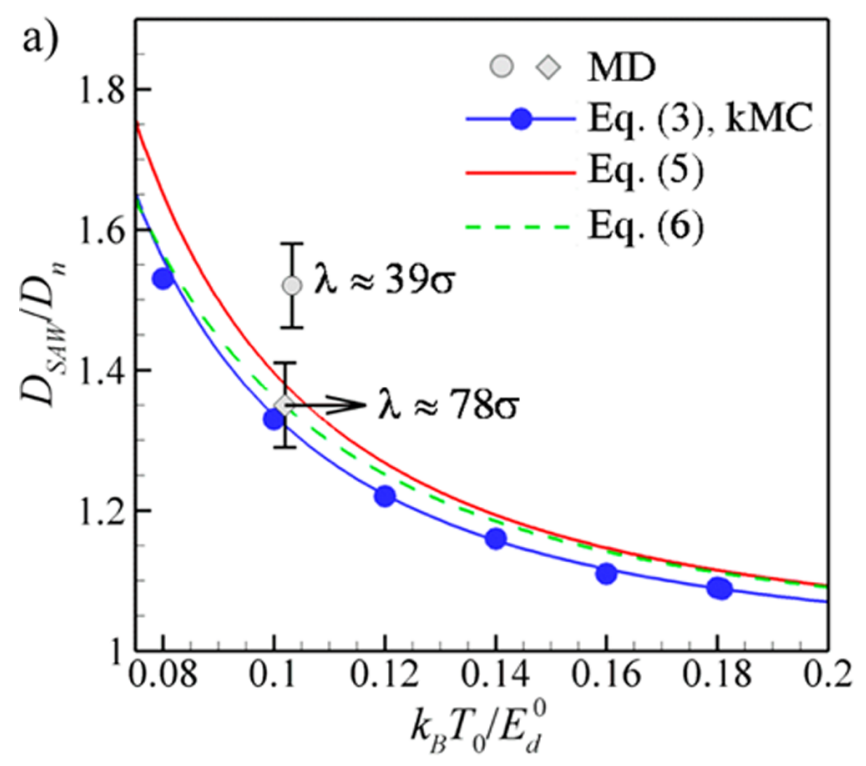

b)

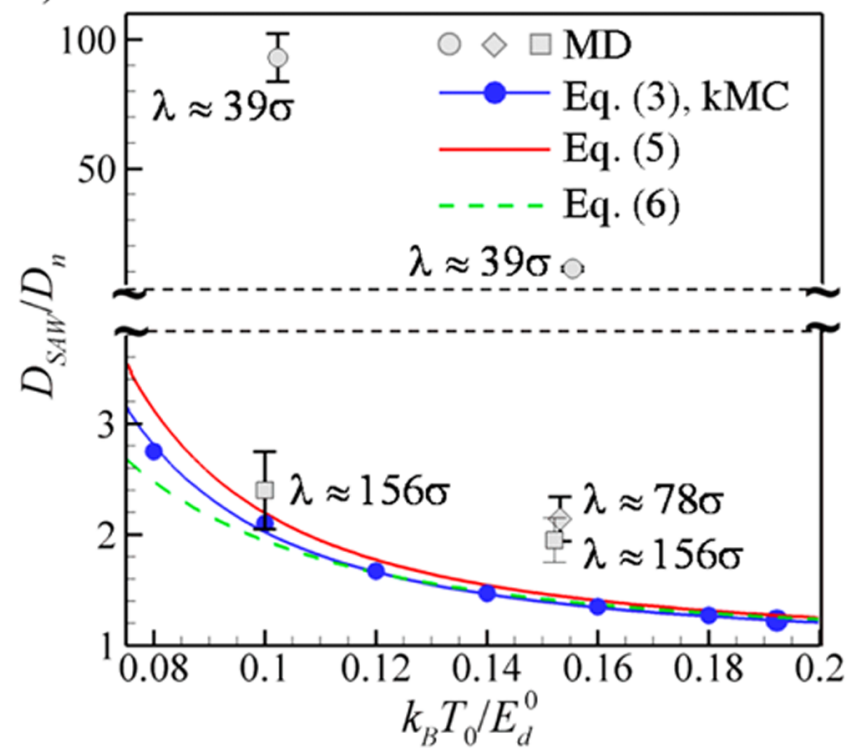

Figure 4. Diffusion enhancement $D_{\mathrm{SAW}} / D_{n}$ by a SAW with $e_{0}=0.048$ as a function of $k_{\mathrm{B}} T_{0} / E_{\mathrm{d}}^{0}$ for adatoms with $\sigma_{\mathrm{a}-\mathrm{s}}=\sigma(\mathrm{a})$ and $\sigma_{\mathrm{a}-\mathrm{s}}=1.5 \sigma$ (b), respectively. The red solid line and the green dashed line show the predictions of eqs 5 and 6 , respectively. The blue solid line and blue circles show the predictions of eq 3 and the results of $\mathrm{kMC}$ calculations, respectively. The large gray symbols show the results of MD simulations, with the error bars corresponding to the standard deviations of the mean calculated based on eight independent series of the simulations.

The MD simulations with the same setup as used above in the analysis of the adiabatic temperature variation and illustrated in Figure 1 are performed for adatoms with $\sigma_{\mathrm{a}-\mathrm{s}}=$ $\sigma$ and $\sigma_{\mathrm{a}-\mathrm{s}}=1.5 \sigma$. For $\sigma_{\mathrm{a}-\mathrm{s}}=\sigma$, the simulations are done for two different wavelengths, $\lambda \approx 39 \sigma$ and $\lambda \approx 78 \sigma$, and the temperature of the thermostat region, $T_{\mathrm{th}}$, maintained at $0.1 E_{\mathrm{d}}^{0} / k_{\mathrm{B}}$. For $\sigma_{\mathrm{a}-\mathrm{s}}=1.5 \sigma$, two series of simulations with $\lambda \approx$ $39 \sigma$ and $\lambda \approx 156 \sigma$ are performed for $T_{\text {th }}=0.1 E_{\mathrm{d}}^{0} / k_{\mathrm{B}}$, and three series of simulations with $\lambda \approx 39 \sigma, \lambda \approx 78 \sigma$, and $\lambda \approx 156 \sigma$ are performed for $T_{\text {th }}=0.152 E_{\mathrm{d}}^{0} / k_{\mathrm{B}}$. Taking the value of the diffusion barrier $E_{\mathrm{d}}^{0}=0.25 \mathrm{eV}$ as an example, the two temperatures used in the MD simulations correspond to 290 
and $441 \mathrm{~K}$. Note that the energy release in the surface region of the substrate due to the dissipation of the SAW results in a slight increase (up to $2 \%$ ) in the steady-state surface temperature above the thermostat temperature $T_{\text {th }}$ in the simulations performed with small $\lambda$. Therefore, the actual surface temperature $T_{0}$, rather than the thermostat temperature, is used in the presentation of the results of the MD simulations. The diffusion coefficients are calculated from the time dependences of the mean square displacements of the adatoms, based on the results of 512 independent trajectories obtained for each set of conditions (except for the simulation with $\sigma_{\mathrm{a}-\mathrm{s}}=$ $1.5 \sigma, T_{\text {th }}=0.1 E_{\mathrm{d}}^{0} / k_{\mathrm{B}}$, and $\lambda \approx 156 \sigma$, for which 1280 simulations were done). The duration of each simulation was $4200 \tau_{0}$ for $T_{\text {th }}$ $=0.1 E_{\mathrm{d}}^{0} / k_{\mathrm{B}}$ and $2100 \tau_{0}$ for $T_{\mathrm{th}}=0.152 E_{\mathrm{d}}^{0} / k_{\mathrm{B}}$.

The values of the diffusion enhancement obtained in MD simulations and shown in Figure 4 demonstrate the convergence to the predictions of the $\mathrm{kMC}$ simulations at long wavelengths of SAWs, thus supporting the discussion of the wavelength-independent strain-induced mechanisms provided above. At the same time, the MD results demonstrate a substantial contribution of the dynamic effects to the diffusion enhancement at small wavelengths, especially for $\sigma_{\mathrm{a}-\mathrm{s}}=1.5 \sigma$. This large contribution of the dynamic effects is surprising given that even for the shortest wavelength $\lambda=39 \sigma$ the period of SAW is $\sim 7.6 \tau_{0}$, several times larger than the period of adatom vibrations, thus excluding the direct resonance coupling between the SAW and adatom vibrations. Spectral analysis of the atomic dynamics in the surface region of the substrate indicates that the generation of higher harmonics is largely suppressed by the dynamic boundary condition designed to maintain the desired shape of the SAW. Nevertheless, the presence of a weak signal from the second (and third in the case of $\lambda \approx 39 \sigma$ ) harmonics leaves the possibility of coupling of the higher harmonics to the adatom vibrations open. The particularly large diffusion enhancement observed for $\sigma_{\mathrm{a}-\mathrm{s}}=$ $1.5 \sigma$ at $\lambda \approx 39 \sigma\left(D_{\mathrm{SAW}} / D_{n}=90 \pm 9\right.$ at $T_{0}=0.102 E_{\mathrm{d}}^{0} / k_{\mathrm{B}}$ and $D_{\mathrm{SAW}} / D_{n}=11.2 \pm 0.8$ at $\left.T_{0}=0.155 E_{\mathrm{d}}^{0} / k_{\mathrm{B}}\right)$, as well as the noticeable contribution of the dynamic effects to the diffusion enhancement even at $\lambda \approx 156 \sigma$ (Figure $4 \mathrm{~b}$ ), can also be related to the relatively long lifetime of the excited vibrational state of the adatom. The lifetime of the excited state is evaluated by exponential fitting of the evolution of the amplitude of the kinetic energy oscillations of an adatom initially placed at a saddle point position on a quenched (zero temperature) substrate, as illustrated in Figure 5. For $\sigma_{\mathrm{a}-\mathrm{s}}=1.5 \sigma$ the lifetime is found to be $\sim 30 \tau_{0}$, which corresponds to about 10 vibrational periods of the adatom and is comparable to the period of SAW with $\lambda \approx 156 \sigma$. The long "memory" of the vibrational excitation is reflecting the weak coupling between the adatom vibrations and substrate phonons and results in frequent multiple jumps (one per 2-3 jumps) observed in the simulations performed for $\sigma_{\mathrm{a}-\mathrm{s}}=1.5 \sigma$. On the contrary, for $\sigma_{\mathrm{a}-\mathrm{s}}$ $=\sigma$ the lifetime of the excited vibrational state of the adatom is only $\sim \tau_{0}$, such that the contribution of the dynamic effects to the diffusion coupling disappears at $\lambda \geq 78 \sigma$ (Figure 4a), and the multiple jumps are rare (e.g., 2 out of 100 jumps at $T_{0}=$ $\left.0.102 E_{\mathrm{d}}^{0} / k_{\mathrm{B}}\right)$.

\section{CONCLUDING REMARKS}

The results of the theoretical analysis and atomistic simulations reported above demonstrate the feasibility of the idea of acoustic activation of surface diffusion and identify the mechanisms responsible for the diffusion enhancement.
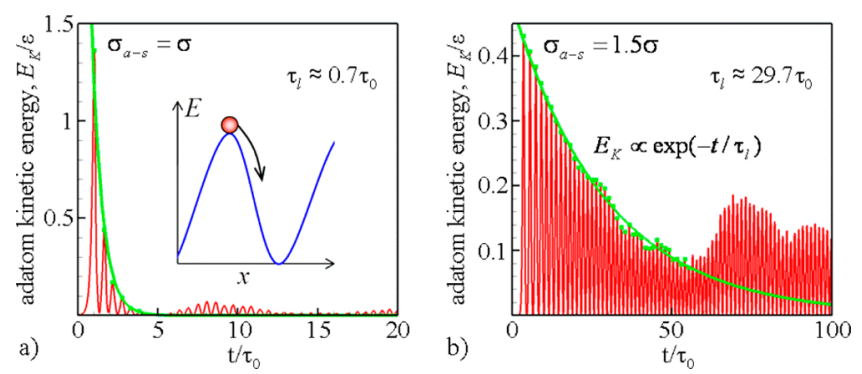

Figure 5. Evaluation of the lifetimes of the excited vibrational state of adatoms with $\sigma_{\mathrm{a}-\mathrm{s}}=\sigma(\mathrm{a})$ and $\sigma_{\mathrm{a}-\mathrm{s}}=1.5 \sigma(\mathrm{b})$. The lifetime is obtained by exponential fitting of the evolution of the amplitude of the oscillations of the kinetic energy an adatom initially placed at a saddle point position on a quenched substrate, as shown schematically in the inset in (a). Green lines show the results of the exponential fitting, with data from the initial parts of the simulations $\left(4 \tau_{0}\right.$ for $\sigma_{\mathrm{a}-\mathrm{s}}=\sigma$ and $54 \tau_{0}$ for $\left.\sigma_{\mathrm{a}-\mathrm{s}}=1.5 \sigma\right)$ used in the fitting. The long-term beatings of the kinetic energy are related to the interaction of weak acoustic pulses generated by the excited adatom with periodic boundary conditions used in the calculations (the substrate represented by $12 \times 12 \times 12 \mathrm{fcc}$ unit cells is used in these calculations).

Transient modification of the diffusion barriers and the reversible/adiabatic temperature variation due to the periodic oscillations of the acoustic strain are shown to be capable of a many-fold enhancement of surface diffusion for systems that exhibit strong strain dependence of the diffusion barriers. Note that the system considered in this work, an adatom on a (001) surface of a LJ crystal, is far from being unique in demonstrating the diffusion enhancement, as the values of $\gamma /$ $E_{\mathrm{d}}^{0}$ reported for various systems can be substantially larger than those listed in Table 1. In particular, a broad range of values of $\gamma / E_{\mathrm{d}}^{0}$ have been reported for biaxially strained substrates, e.g., 8.6 for $\mathrm{Ag} / \mathrm{Ag}(111),{ }^{23}-5$ for $\mathrm{Ge} / \mathrm{Si}(001),{ }^{27}-15$ for $\mathrm{Ge} /$ $\mathrm{Ge}(001),{ }^{27}-2.7$ for $\mathrm{In} / \mathrm{GaAs}(001),{ }^{25}$ and from -6.8 to 3.4 (for different diffusion paths) for $\mathrm{Si} / \mathrm{Si}(001){ }^{26}$ The value of $\gamma$ calculated in our work for the uniaxially strained substrate with $\sigma_{\mathrm{a}-\mathrm{s}}=\sigma$ is about two times smaller than the value reported for the same system under conditions of biaxial strain, ${ }^{24}$ suggesting that, in accord with similar observations for $\mathrm{Si}(001)$ surface, ${ }^{26}$ the components of surface strain make a near-additive contribution to the scaling of the diffusion barrier.

An approximate scaling law established in the theoretical analysis reveals the connections between the diffusion enhancement due to the transient variation of the acoustic strain and the parameters characterizing the acoustic wave and surfaceadatom interaction. Importantly, the scaling law predicts independence of the strain-induced enhancement on the frequency of the SAW. An additional mechanism of acoustic activation of surface diffusion that does exhibit strong frequency dependence is the dynamic coupling of the SAW to the vibrational states of the adsorbed species. More than an order of magnitude diffusion enhancement is observed in $\mathrm{MD}$ simulations when the frequency of the SAW is about 3 times lower than the vibrational frequency of the adsorbate. While the contribution of the dynamic coupling to the diffusion enhancement diminishes with decreasing frequency of the SAW, the presence of this effect down to frequencies that are an order of magnitude lower than the vibrational frequency of the adsorbate, combined with the large magnitude of the dynamic diffusion enhancement, suggest the potential practical importance of this mechanism in applications utilizing highfrequency SAWs in the range of tens of gigahertz. ${ }^{19-21}$ 
Overall, the results of this study reveal the fundamental physical mechanisms of acoustic activation of surface diffusion and establish the dependence of the surface processes on the parameters of the acoustic waves. The acoustic activation of surface processes can serve as an attractive alternative to the conventional thermal activation in thin film growth on heatsensitive substrates, and the mechanistic insights obtained in this study provide guidelines for the design of novel film growth techniques.

\section{ASSOCIATED CONTENT}

\section{S Supporting Information}

Three movies are provided as Supporting Information. Movies S1 and S2 (jp400884d_si_002.avi and jp400884d_si_003.avi) supplement the strain and temperature profiles shown in Figure 2a. Movie S3 (jp400884d_si_004.avi) shows a typical trajectory of an adatom in a MD simulation used in the analysis of surface diffusion in the presence of a SAW. This material is available free of charge via the Internet at http://pubs.acs.org.

\section{AUTHOR INFORMATION}

\section{Corresponding Author}

*Tel.: + (434) 243-3582. E-mail: lz2n@virginia.edu.

\section{Notes}

The authors declare no competing financial interest.

\section{ACKNOWLEDGMENTS}

The financial support of this work is provided by DARPA. Computational support is provided by NSF/XSEDE (TGDMR110090) and OLCF (MAT048). The authors would like to thank Dr. Henry Helvajian of The Aerospace Corporation, Dr. Anne E. Fischer of the Strategic Analysis, Inc., and Dr. Brian Holloway of DARPA for insightful and stimulating discussions.

\section{REFERENCES}

(1) Modern Acoustical Techniques for the Measurement of Mechanical Properties; Levy, M., Bass, H. E., Stern, R., Eds.; Academic: New York, 2001.

(2) Kozhushko, V. V.; Lomonosov, A. M.; Hess, P. Intrinsic Strength of Silicon Crystals in Pure- and Combined-Mode Fracture without Precrack. Phys. Rev. Lett. 2007, 98, 195505.

(3) Lomonosov, A. M.; Grigoriev, P. V.; Hess, P. Sizing of Partially Closed Surface-Breaking Microcracks with Broadband Rayleigh Waves. J. Appl. Phys. 2009, 105, 084906.

(4) Wood, C. D.; Evans, S. D.; Cunningham, J. E.; O’Rorke, R.; Wälti, C.; Davies, A. G. Alignment of Particles in Microfluidic Systems Using Standing Surface Acoustic Waves. Appl. Phys. Lett. 2008, 92, 044104.

(5) Hennig, M.; Neumann, J.; Wixforth, A.; Radler, J. O.; Schneider, M. F. Dynamic Patterns in a Supported Lipid Bilayer Driven by Standing Surface Acoustic Waves. Lab Chip 2009, 9, 3050-3053.

(6) Inoue, Y.; Matsukawa, Y.; Sato, K. Effect of Surface Acoustic Wave Generated on Ferroelectric Support upon Catalysis. J. Am. Chem. Soc. 1989, 111, 8965-8966.

(7) Watanabe, Y.; Inoue, Y.; Sato, K. Activation of a Thin Film Pd Catalyst for Co and Ethanol Oxidation by Surface Acoustic Waves. Surf. Sci. 1996, 357-358, 769-772.

(8) Kelling, S.; Mitrelias, T.; Matsumoto, Y.; Ostanin, V. P.; King, D. A. Acoustic Wave Enhancement of the Catalytic Oxidation of Carbon Monoxide over Pt $\{110\}$. J. Chem. Phys. 1997, 107, 5609-5612.

(9) Kelling, S.; Cerasari, S.; Rotermund, H. H.; Ertl, G.; King, D. A. A Photoemission Electron Microscopy (PEEM) Study of the Effect of Surface Acoustic Waves on Catalytic CO Oxidation over Pt $\{110\}$. Chem. Phys. Lett. 1998, 293, 325-330.
(10) Saito, N.; Nishiyama, H.; Inoue, Y. Acoustic Wave Effects on Catalysis: Design of Surfaces with Artificially Controllable Functions for Chemical Reactions. Appl. Surf. Sci. 2001, 169-170, 259-263.

(11) Inoue, Y. Effects of Acoustic Waves-Induced Dynamic Lattice Distortion on Catalytic and Adsorptive Properties of Metal, Alloy and Metal Oxide Surfaces. Surf. Sci. Rep. 2007, 62, 305-336.

(12) Lindner, B.; Seydel, U. Laser Desorption Mass Spectrometry of Nonvolatiles under Shock Wave Conditions. Anal. Chem. 1985, 57, 895-899.

(13) Basov, N. G.; Belenov, E. M.; Gubin, M. A.; Kurdoglyan, M. S.; Nikitin, V. V.; Oraevskii, A. N.; Chichkov, B. N. New Ways of Obtaining Cold Atoms and Molecules, Sov. J. Quantum Electron. 1987, 17, 919-922.

(14) Golovlev, V. V.; Allman, S. L.; Garrett, W. R.; Chen, C. H. Laser-Induced Acoustic Desorption of Electrons and Ions. Appl. Phys. Lett. 1997, 71, 852-854.

(15) Golovlev, V. V.; Allman, S. L.; Garrett, W. R.; Taranenko, N. I.; Chen, C. H. Laser-Induced Acoustic Desorption. Int. J. Mass Spectrom. 1997, 169, 69-78.

(16) Zinovev, A. V.; Veryovkin, I. V.; Moore, J. F.; Pellin, M. J. LaserDriven Acoustic Desorption of Organic Molecules from BackIrradiated Solid Foils. Anal. Chem. 2007, 79, 8232-8241.

(17) Dow, A. M.; Wittrig, A. R.; Kenttämaa, H. I. Laser-Induced Acoustic Desorption (LIAD) Mass Spectrometry. Eur. J. Mass Spectrom. 2012, 18, 77-92.

(18) Helvajian, H., private communication, 2013

(19) Siemens, M. E.; Li, Q.; Murnane, M. M.; Kapteyn, H. C.; Yang, R.; Anderson, E. H.; Nelson, K. A. High-Frequency Surface Acoustic Wave Propagation in Nanostructures Characterized by Coherent Extreme Ultraviolet Beams. Appl. Phys. Lett. 2009, 94, 093103.

(20) Schubert, M.; Grossmann, M.; Ristow, O.; Hettich, M.; Bruchhausen, A.; Barretto, E. C. S.; Scheer, E.; Gusev, V.; Dekorsy, T. Spatial-Temporally Resolved High-Frequency Surface Acoustic Waves on Silicon Investigated by Femtosecond Spectroscopy. Appl. Phys. Lett. 2012, 101, 013108.

(21) Nardi, D.; Zagato, E.; Ferrini, G.; Giannetti, C.; Banfi, F. Design of a Surface Acoustic Wave Mass Sensor in the $100 \mathrm{GHz}$ Range. Appl. Phys. Lett. 2012, 100, 253106.

(22) Roland, C.; Gilmer, G. H. Epitaxy on Surfaces Vicinal to $\mathrm{Si}(001)$. I. Diffusion of Silicon Adatoms over the Terraces. Phys. Rev. B 1992, 46, 13428-13436.

(23) Ratsch, C.; Seitsonen, A. P.; Scheffler, M. Strain Dependence of Surface Diffusion: $\mathrm{Ag}$ on $\mathrm{Ag}(111)$ and $\mathrm{Pt}(111)$. Phys. Rev. B 1997, 55, 6750-6753.

(24) Schroeder, M.; Wolf, D. E. Diffusion on Strained Surfaces. Surf. Sci. 1997, 375, 129-140.

(25) Penev, E.; Kratzer, P.; Scheffler, M. Effect of Strain on Surface Diffusion in Semiconductor Heteroepitaxy. Phys. Rev. B 2001, 64, 085401.

(26) Shu, D. J.; Liu, F.; Gong, X. G. Simple Generic Method for Predicting the Effect of Strain on Surface Diffusion. Phys. Rev. B 2001, 64,245410

(27) van de Walle, A.; Asta, M.; Voorhees, P. W. First-Principles Calculation of the Effect of Strain on the Diffusion of Ge Adatoms on $\mathrm{Si}$ and Ge(001) Surfaces. Phys. Rev. B 2003, 67, 041308.

(28) Stoddard, S. D.; Ford, J. Numerical Experiments on the Stochastic Behavior of a Lennard-Jones Gas System. Phys. Rev. A 1973, $8,1504-1512$.

(29) Landau, L. D.; Lifshitz, E. M. Theory of Elasticity, 3rd ed.; Pergamon Press: Oxford, 1986.

(30) Stoneley, R. The Propagation of Surface Elastic Waves in a Cubic Crystal. Proc. R. Soc., London A 1955, 232, 447-458.

(31) Berendsen, H. J. C.; Postma, J. P. M.; van Gunsteren, W. F.; DiNola, A.; Haak, J. R. Molecular Dynamics With Coupling to an External Bath. J. Chem. Phys. 1984, 81, 3684-3690. 